\title{
ALL $n$-UNIFORM QUASITRANSLATION HJELMSLEV PLANES ARE STRONGLY $n$-UNIFORM ${ }^{1}$
}

\section{DAVID A. DRAKE}

ABSTRACT. An affine $H$-plane $Q$ is called a quasitranslation $H$-plane if it possesses a group of quasitranslations which is regular on the points of $Q$. Let $n$ be any positive integer. Then every $n$-uniform quasitranslation $H$-plane is strongly $n$-uniform.

Hjelmslev planes of level $n, n$ an arbitrary integer, have been studied by Artmann and the author. Among other results, the author has proved [2, Theorem 5.4] that all finite Desarguesian $H$-planes are of level $n$ for some $n$, a result later generalized by Artmann [1] to include all Desarguesian $H$ planes whose coordinatizing rings have nilpotent radicals. An $H$-plane is strongly $n$-uniform if it is both finite and of level $n$. There exist recent unpublished examples of finite translation $H$-planes which are strongly $n$ uniform for no $n$; however, we shall prove that all (finite) translation $H$ planes which are $n$-uniform are indeed strongly $n$-uniform. This theorem greatly generalizes a result appearing in [3, Corollary 4.2]. The proof of the theorem is elementary, involving little more than counting arguments on some subgroups of finite abelian groups.

Reversing the author's usual notational practice, lower case letters will be used throughout the paper to denote points; capitals, to denote lines. With this exception, all notation and definitions will coincide with the usage in [3]. In the interest of brevity, the notation, definitions and statements of theorems from [3, pp. 252-256] will not be repeated in this paper. We shall write $[3,1, i]$ rather than [3, Proposition 1.1(i)]. The frequently quoted $[3,1,6]$ contains a compositor's error: $(\simeq i)$ should be replaced by $(\sim i)$.

Let $\mathfrak{Q}$ be an affine $H$-plane (an $A H$-plane); $\theta$, a mapping of the point set of $\mathscr{A}$ into itself. One calls $\theta$ a dilatation if to each line $G$ of $\mathfrak{A}$ corresponds a

Received by the editors October 27, 1973 and, in revised form, May 24, 1974. AMS (MOS) subject classifications (1970). Primary 05B25; Secondary 05B30, 50A20.

Key words and phrases. Hjelmslev plane, level $n, n$-uniform, strongly $n$-uniform, translation ( $H$-plane), quasitranslation ( $H$-plane).

1 This research was partially supported by National Science Foundation Grant GP-39059. 
parallel line $G^{*}$ such that $\theta(G) \subset G^{*}$. A dilatation is called a quasitranslation if it is the identity map or is fixed point free. An $A H$-plane is called a quasitranslation $H$-plane if it possesses a regular group of quasitranslations.

Theorem (Luineburg). An AH-plane is a quasitranslation H-plane if and only if it is isomorphic (as incidence structure with parallelism) to some $J(T, \pi)$. The quasitranslations are the right multiplications by elements of $T$.

The proof that an $A H$-plane representable by some $J(T, \pi)$ is a quasitranslation $H$-plane is given in [4, p. 274]. A sketch of a proof of the converse is also given in [4]. (See the proof of Satz 4.3.)

Definition. Let $\mathfrak{Q}$ be an $n$-uniform $A H$-plane. Then we say that $\mathfrak{A}$ satisfies Axiom $\mathrm{S}$ or that $\mathfrak{Q}$ has Property $\mathrm{S}$ (called Property $\mathrm{A}$ in [2]) provided that the following assertion is true. Whenever $p \in H \cap K,|H \cap K|=$ $r^{i}, q \in H-K, p(\simeq j) q$, and $i+j<n$, it follows that $q(\simeq i+j) K$. An $A H-$ plane is said to be strongly $n$-uniform if it is $n$-uniform and has Property $S$.

We are now prepared to state and prove

Theorem. Every (finite) n-uniform quasitranslation AH-plane is strongly n-uniform.

Proof. Let $\mathfrak{Q}=J(T, \pi)$ be an arbitrary $n$-uniform quasitranslation $A H$ plane with invariant $r$. We shall use additive notation for $T$, and, in particular, we shall denote the identity of $T$ by 0 . For $0 \leq i \leq n$, we write $T^{i}$ to denote $\{x \in T: x(\sim n-i) 0\}$. Let $x$ and $y$ be arbitrary elements of $T$. It follows easily from the definitions of $(\sim i)$ and $J(T, \pi)$ that

$$
x(\sim i) y \Leftrightarrow y-x \in T^{n-i}, \quad 0 \leq i \leq n .
$$

Next, let $x$ and $y$ be elements of $T^{i}$. Then $(y+x)-x=y \in T^{i}$. It follows from (1) that $x(\sim n-i)(y+x)$. Since $x(\sim n-i) 0,[3,1,6]$ implies that $(y+x)(\sim n-i) 0$; i.e., that $y+x \in T^{i}$. Since $T$ is finite, we have proved that

$$
T^{i} \text { is a subgroup of } T \text { for } 0 \leq i \leq n \text {. }
$$

For each point $b$ in $T$ we define a map $\theta^{b}$ on the points of $T$ by the rule: $\theta^{b}(x)=x+b$ for all $x \in T$. Clearly, the $\theta^{b}$ are a group of incidence structure automorphisms (quasitranslations indeed!) of $\mathbb{A}$ which are transitive on the points of $\mathbb{A}$. To prove the theorem then, it suffices to establish that Axiom $S$ holds when $p$ is equal to 0 . Let $p=0, q, H$ and $K$ be points and lines of $\mathfrak{Q}$ which satisfy the hypotheses of Axiom $S$. Assume first that 
$j=n-1 .[3,1,3]$ and the necessity that $i+j$ be less than $n$ jointly imply that $i=0$. It follows from $[3,1,2]$ and the fact that $q \notin K$ that $q(\mathcal{L} n) K$. Then $q(\simeq n-1) K$. We have established that Axiom S holds when $j=n-1$.

Proceeding by reverse induction on $j$, we now assume that $0 \leq j<n-1$. Since $p=0 \in H \cap K$, it follows that $H, K \in \pi$. Now if $G$ is an arbitrary element of $\pi$, we set

$$
\begin{aligned}
G^{\prime}=G \cap T^{n-j}, \quad G^{\#} & =G \cap\left(T^{n-j}-T^{n-j-i}\right), \\
G^{\prime}(x)=T^{n-x-j-1}+G^{\prime}, \quad G^{\#}(x) & =T^{n-x-j-1}+G^{\#} .
\end{aligned}
$$

Let $G$ and $L$ be elements of $\pi$ such that $|G \cap L|=r^{x}, x \leq i$. It follows from $[3,1,4]$ and $[3,1,8]$ that $\left|L^{\prime}\right|=r^{n-j},\left|L^{\prime} \cap T^{n-x-j-1}\right|=r^{n-x-j-1}$, and $\left|T^{n-x-j-1}\right|=r^{2 n-2 x-2 j-2}$. Then $\left|L^{\prime}(x)\right|=r^{2 n-x-2 j-1}$; consequently,

$$
\left|G^{\prime}+L^{\prime}(x)\right|=\left(r^{3 n-x-3 j-1}\right) /\left|G^{\prime} \cap L^{\prime}(x)\right| \text {. }
$$

Let $b \in G \cap T^{n-j-1}$. If $b \in L$, it is clear that $b \in L^{\prime}(x), G^{\prime}$. Consider then the case that $b \notin L$. In this case, it follows from $[3,1,2]$ and $[3,1,9]$ that $b(\simeq j+m) 0$ for some positive integer $m$ which satisfies $j+m<n-x$. The induction assumption on $j$ thus implies that $b(\simeq x+j+m) L$. This means that there is a point $c$ on $L$ such that $c(\sim x+j+1) b$. It follows from $[3,1,6]$ that $c \in T^{n-j-1}$; hence $c \in L^{\prime}$. By $(1), b-c \in T^{n-x-j-1}$. Then $b=(b-c)+c$ is in $L^{\prime}(x)$. We have established that

$$
G \cap T^{n-j-1} \subset L^{\prime}(x) \cap G^{\prime} \text {. }
$$

Since $|G \cap L|=r^{x}$ and since $x \leq i<n-j,[3,1,9]$ implies that $G \cap L=$ $G \cap T^{x}=G^{\prime} \cap L^{\prime}$. Then $\left|G^{\prime} \cap L^{\prime}\right|=r^{x}$; consequently

$$
\left|G^{\prime}+L^{\prime}(x)\right| \geq\left|G^{\prime}+L^{\prime}\right|=r^{2 n-x-2 j} \text {. }
$$

Now assume that there is a point $b$ in $\left(G^{\prime} \cap L^{\prime}(x)\right)-T^{n-j-1}$. Then (4) and $[3,1,4]$ imply that $\left|G^{\prime} \cap L^{\prime}(x)\right|>r^{n-j-1}$; substitution of the last inequality into (3) yields a contradiction of (5). We have established that

$$
\left|G^{\#} \cap L^{\prime}(x)\right|=0
$$

if $|G \cap L|=r^{x}$ and $x \leq i$.

Now let $L$ be an arbitrary element of $\pi, 0 \leq x \leq i$, and let $b$ be an arbitrary element of $L^{\#}(x)$. Then $b=(b-c)+c$ where $b-c \in T^{n-x-j-1}$ and $c \in L^{\#}$. By $(1), b(\sim x+j+1) c$. By definition of $L^{\#}, c(\simeq j) 0$. Then $[3,1,6]$ implies that $b(\sim j) 0$. We have proved that

$$
L^{\#}(x) \subset T^{n-j}-T^{n-j-1}
$$

when $L \in \pi$ and $0 \leq x \leq i$. 
Next, let $G$ be an element of $\pi$ such that $|G \cap H|=r^{x}, x \leq i$. Assume that there exists a point $b$ in $G \cap H^{\#}(x)$. Then $b \in H^{\prime}(x)$, and (7) implies that $b \in T^{n-j}-T^{n-j-1}$, hence, that $b \in G^{\#}$. The resulting contradiction of (6) yields the result that $\left|G \cap H^{\#}(x)\right|=0$ whenever $|G \cap H|=r^{x}, x \leq i$. Since $G \cap H^{\#}(x) \subset G \cap H^{\#}(y)$ whenever $y \leq x$, we have established that

$$
\left|G \cap H^{\#}(x)\right|=0
$$

whenever $|G \cap H|=r^{y}, y \leq x \leq i$. Let $a \in T^{n-x-j-1}, b \in H^{\#}, c \in D=H \cap$ $T^{n-x-j-1}$. The element $a+b$ of $H^{\#}(x)$ may be represented as $(a-c)+$ $(c+b)$. It follows from (2) that $a-c \in T^{n-x-j-1}$; from $(1)$ and $[3,1,6]$, that $c+b \in H^{\#}$. Then each element of $H^{\#}(x)$ has at least $|D|$ representations of the form $a+b$ with $a \in T^{n-x-j-1}$ and $b \in H^{\#}$. Next, let $a+b=$ $a^{\prime}+b^{\prime}$ be two such representations. Then $-a^{\prime}+a=b^{\prime}-b \in D$. For a given $b$, there exist at most $|D|$ choices for $b^{\prime}$ such that $b^{\prime}-b \in D$. Then each element of $H^{\#}(x)$ has at most and hence precisely $|D|$ representations of the form $a+b$ with $a \in T^{n-x-j-1}$ and $b \in H^{\#}$. It follows from $[3,1,4]$ and $[3,1,8]$ that

$$
\left|H^{\#}(x)\right|=\left(r^{n-j}-r^{n-j-1}\right) \cdot r^{n-x-j-1} \text {. }
$$

Since $T$ is covered by the components of $\pi$, (7) implies that $H^{\#}(x)$ is covered by $\left\{G^{\#}: G \in \pi\right\}$. It follows from $[3,1,4]$ that

$$
\left|G^{\#}\right|=r^{n-j}-r^{n-j-1}
$$

for each $G \in \pi$. Then (9) implies that the number of distinct nonempty sets of the form $H^{\#}(x) \cap G^{\#}$ is at least $r^{n-x-j-1}$. It follows from $[3,1,3]$ and $[3,1,9]$ that the nonempty set $H^{\#}(x) \cap G^{\#}$ equals the set $H^{\#}(x) \cap L^{\#}$ if and only if $G^{\prime}=L^{\prime}$. It follows from $[3,1,3],[3,1,5]$ and $[3,1,9]$ that the number of components $L$ with $L^{\prime}=G^{\prime}$ for a fixed $G$ is $r^{j}$. Then the number of $G$ in $\pi$ for which $H^{\#}(x) \cap G$ is nonempty is at least $r^{n-x-1} .[3,1,5]$ asserts that the number of $G$ in $\pi$ for which $|G \cap H| \geq r^{x+1}$ is precisely $r^{n-x-1}$. By (8) and $[3,1,3]$, every $G$ in $\pi$ which satisfies $\left|H^{\#}(x) \cap G^{\#}\right|>0$ must also satisfy $|G \cap H| \geq r^{x+1}$. It follows that

$$
\left|H^{\#}(x) \cap G^{\#}\right|>0 \Leftrightarrow|G \cap H| \geq r^{x+1} \text {. }
$$

We have also established that the number of $G$ in $\pi$ for which $\left|H^{\#}(x) \cap G^{\#}\right| \neq$ 0 is precisely $r^{n-x-1}$. Then the number of distinct nonempty sets $H^{\#}(x) \cap$ $G^{\#}$ with $G$ in $\pi$ is precisely $r^{n-x-j-1}$. Since $H^{\#}(x)$ is covered by the sets 
$H^{\#}(x) \cap G^{\#}$, assertions (9), (10) and (11) imply that

$$
\left|H^{\#}(x) \cap G^{\#}\right|=r^{n-j}-r^{n-j-1} \Leftrightarrow|G \cap H| \geq r^{x+1} \text {. }
$$

Substituting $K$ for $G$ and $i-1$ for $x$ in (12) yields the conclusion that

$$
\left|\left(T^{n-i-j}+H^{\#}\right) \cap K^{\#}\right|=r^{n-j}-r^{n-j-1} \text {. }
$$

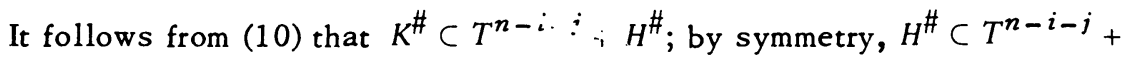
$K^{\#}$. Recall that $p=0, q, H$ and $K$ are points and lines which satisfy the hypotheses of Axiom S. Then $q \in H^{\#}$. It follows that $q=(q-b)+b$ for some $b \in K^{\#}, q-b \in T^{n-i-j}$. Then (1) implies that $q(\sim i+j) K$. Suppose that $q(\sim i+j+1) c$ for some $c \in K$. Then (1) implies that $c \in T^{n-i-j-1}+$ $q \subset H^{\#}(i)$. Using (7) with $x=i$, we conclude that $c \in K^{\#}$. The resulting contradiction of (11) forces the conclusion that $q(\mathcal{L} i+j+1) K$. Then $q(\simeq i+j) K$. This is the desired conclusion. The induction on $j$ is complete, and hence the theorem is proved.

\section{REFERENCES}

1. B. Artmann, Desarguessche Hjelmslev-Ebenen n-ter Stufe, Mitt. Math. Sem. Giessen 91 (1971), 1-19. MR 45 \#4270.

2. D. A. Drake, On n-uniform Hjelmslev planes, J. Combinatorial Theory 9 (1970), 267-288. MR $42 \# 3667$.

3. - The structure of n-uniform translation Hjelmslev planes, Trans. Amer. Math. Soc. 175 (1973), 249-282. MR 46 \#9853.

4. H. Lüneburg, Affine Hjelmslev-Ebenen mit transitiver Translationsgruppe, Math. Z. 79 (1962), 260-288. MR 25 \#1479.

DEPARTMENT OF MATHEMATICS, UNIVERSITY OF FLORIDA, GAINESVILLE, FLORIDA 32611 